\title{
LATERAL WAVES IN ANISOTROPIC OPTICAL WAVEGUIDES
}

\author{
M. WABIA \\ Institute of Physics, Technical University of Szczecin \\ Al. Piastów 48, 70-310 Szczecin, Poland \\ (Received June 24, 1991; revised version March 24, 1992) \\ The paper deals with the properties of lateral waves in uniaxial optical \\ waveguides. The lateral waves are closely associated with the phenomenon \\ of the total reflection and arise due to the presence of branch point singu- \\ larities of the reflection coefficients. In the structure of uniaxial asymmetric \\ waveguides two sets of the lateral waves may propagate along two interfaces \\ of the waveguide. A detailed wave-optical analysis for the propagation is
} presented.

PACS numbers: $42.80 . L t$

\section{Introduction}

The field emitted by a source in the presence of an interface comprises, as well known, a few components. Apart from the contributions from incident and reflected waves constituting the so-called geometric-optical field we have to deal also with diffractional contributions. The diffractional field is given, most frequently, in the form of surface or leaky waves, which can be guided by the interface [1-5]. An important contribution to the diffractional field is given also by the so-called lateral wave $[1,5,8]$.

When an electromagnetic wave falls on an interface between two isotropic dielectrics, then on both sides of the interface secondary fields appear (reflected and refracted ones). If the field impinges from the denser medium, the refracted wave emerges at a steeper angle with respect to the normal to the interface, and for incidence at the critical angle, refraction is parallel to the boundary. The lateral wave is just connected with this refracted field. It is a wave type that is supported by the simplest possible boundary configuration, namely, the planar interface between two dielectric half spaces. However, while the energy of the lateral wave is guided along this boundary, a portion of its flux radiates away at the critical angle of total reflection. Hence, if a lateral wave is set up at some point of the 
interface, its field progresses along the interface, but the continuous energy leakage ultimately reduces its amplitude [1].

The rapid development of integrated optics utilizes electromagnetic waves of the inhomogeneous type, which include lateral, surface and leaky waves. Whereas these waves have been widely used in the area of radiowave technology, particularly in the microwave range, their application to optical devices is new [1-8].

The above-mentioned inhomogeneous waves constitute, in the field of the modern integrated optics, a basis for operation of many devices. This justifies the studies of properties of inhomogeneous waves, among others, also in a fundamental structure in the integrated optics such as an optical waveguide.

Surface and leaky waves in the waveguide structure have been discussed in the literature $[2-4,9,10]$. The present paper is devoted to lateral waves. The investigation carried out on lateral waves have a particular importance due to their essential role in the propagation model of the electromagnetic field in the waveguide, i.e., in the "zigzag" model.

A possibility of guidance of lateral waves by a dielectric layer on a metallic substratum has been considered by Tamir and Felsen [5]. In this case the total internal reflection occurs only on one interface between media and the lateral wave guided by the layer does not essentially differ from the wave guided by a single boundary. In the present paper we shall analyse the possibility of the guidance of lateral waves in an uniaxial optical waveguide, i.e., in the system with two interfaces on which the total internal reflection occurs. The optical anisotropic waveguide has been chosen for the analysis because of its richer physical substance compared with that of an isotropic one.

A generalization of the propagation model for the electromagnetic field in an optical waveguide - the "zigzag" model will be proposed by placing a lateral wave in it.

In order to realize the problem put forward we shall use the spectral representation of the electromagnetic field in an uniaxial planar waveguide excited by a linear source [10].

\section{Formulation of the problem}

The anisotropic waveguide considered here constitutes a two-dimensional uniaxial structure with three anisotropic layers. The geometry of the system is shown in Fig. 1. The thickness of the film is $2 l$ and the direction of the optical axis of each layer is determined by the direction of a unit vector $c$. The relative permittivity tensors in the $j$-region $(j=1,2,3)$ of the guide, corresponding to the optical axis in the $x, z$-plane, are all of the form

$$
{ }^{j} \varepsilon=\left[\begin{array}{ccc}
{ }^{j} \varepsilon_{11} & 0 & { }^{j} \varepsilon_{13} \\
0 & { }^{j} \varepsilon_{22} & 0 \\
{ }^{j} \varepsilon_{31} & 0 & { }^{j} \varepsilon_{33}
\end{array}\right]
$$

with

$$
\varepsilon_{i j}=\varepsilon^{o}\left(\delta_{i j}-c_{i} c_{j}\right)+\varepsilon^{\mathbf{e}} c_{i} c_{j},
$$




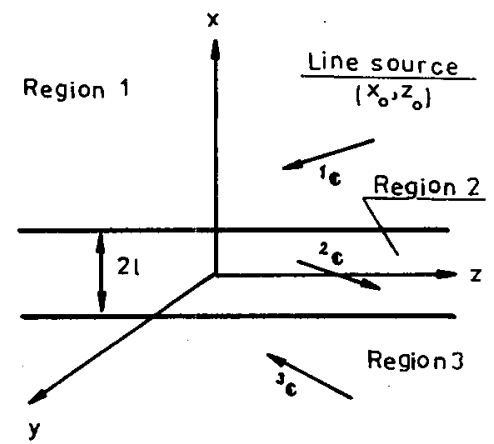

Fig. 1. Geometry of the source-excited uniaxial waveguide. ${ }^{1} c,{ }^{2} c$ and ${ }^{3} c$ are the unit vectors in the directions of the optical axis of layers, respectively.

where $\varepsilon^{o}$ and $\varepsilon^{\mathrm{e}}$ are the ordinary and extraordinary relative permittivities, respectively. The harmonically oscillating magnetic line excitation source is located at point $x=x_{0}, z=z_{0}$ in the top layer.

For simplicity only the TM-type field will be considered here. As it is well-known [10], the TM wave propagating along the $z$ direction has a single component of the magnetic field $H_{y}$, from which the electric field components of $E_{x}$ and $E_{z}$ in regions $j=1,2,3$ may be found

$$
\begin{aligned}
& { }^{j} E_{x}=-\mathrm{i} \omega^{-1} \varepsilon_{0}^{-1} \alpha_{j}^{-1}\left({ }^{j} \varepsilon_{13} \partial^{j} H_{y} / \partial x+{ }^{j} \varepsilon_{33} \partial^{j} H_{y} / \partial z\right), \\
& { }^{j} E_{z}=-\mathrm{i} \omega \varepsilon_{0}^{-1} \alpha_{j}^{-1}\left({ }^{j} \varepsilon_{11} \partial^{j} H_{y} / \partial x+{ }^{j} \varepsilon_{13} \partial^{j} H_{y} / \partial z\right),
\end{aligned}
$$

where

$$
\alpha_{j}=\left({ }^{j} \varepsilon_{11}{ }^{j} \varepsilon_{33}-{ }^{j} \varepsilon_{13}^{2}\right) .
$$

The integral representation of $H_{y}$ has been found for all regions of the waveguide structure [10]. In the region of interest here (the film) it has the form

$$
\begin{aligned}
& { }^{2} H_{y}(x, z)=\frac{1}{4 \pi} \int_{-\infty}^{+\infty} \gamma_{2}^{-1} g_{2+}(h) \exp \left[{ }^{2} \varepsilon_{11}^{-1}\left(\gamma_{2}-\mathrm{i}^{2} \varepsilon_{13} h\right) x-\mathrm{i} h z\right] \mathrm{d} h \\
& +\frac{1}{4 \pi} \int_{-\infty}^{+\infty} \gamma_{2}^{-1} g_{2-}(h) \exp \left[{ }^{2} \varepsilon_{11}^{-1}\left(\gamma_{2}+\mathrm{i}^{2} \varepsilon_{13} h\right) x-\mathrm{i} h z\right] \mathrm{d} h,
\end{aligned}
$$

where $h$ is the propagation constant in the $z$ direction,

$$
\begin{aligned}
& k_{0}=\left(\mu_{0} \varepsilon_{0}\right)^{1 / 2}, \quad \gamma_{j}=\left[\alpha_{j}\left(h^{2}-k_{j}^{2}\right)\right]^{1 / 2}, \quad k_{j}=k_{0}\left({ }^{j} \varepsilon_{11}\right)^{1 / 2}, \\
& g_{2+}(h)=q(c+d)^{-1} \exp \left[{ }^{1} \varepsilon_{11}^{-1}\left(\gamma_{1}+\mathrm{i}{ }^{1} \varepsilon_{13} h\right)\left(l-x_{0}\right)\right. \\
& \left.-{ }^{1} \varepsilon_{11}^{-1}\left(\gamma_{2}+\mathrm{i}{ }^{2} \varepsilon_{13} h\right)\right] \\
& g_{2-}(h)=f(c+d)^{-1} \exp \left[{ }^{1} \varepsilon_{11}^{-1}\left(\gamma_{1}+\mathrm{i}^{1} \varepsilon_{13} h\right)\left(l-x_{0}\right)\right. \\
& \left.+{ }^{1} \varepsilon_{11}^{-1}\left(\gamma_{2}-\mathrm{i}{ }^{2} \varepsilon_{13} h\right)\right]
\end{aligned}
$$




$$
\begin{aligned}
& c=\left(\alpha_{2}^{2} \gamma_{1} \gamma_{2}+\alpha_{1} \alpha_{3} \gamma_{2}^{2}\right) \operatorname{sh}\left(2{ }^{2} \varepsilon_{11}^{-1} \gamma_{2} l\right), \\
& d=\left(\alpha_{2} \gamma_{2}\right)\left(\alpha_{1} \gamma_{3}+\alpha_{3} \gamma_{1}\right) \operatorname{ch}\left(2{ }^{2} \varepsilon_{11}^{-1} \gamma_{2} l\right), \\
& q=-\alpha_{2} \gamma_{2}\left(\alpha_{2} \gamma_{3}-\alpha_{3} \gamma_{2}\right), \\
& f=\alpha_{2} \gamma_{2}\left(\alpha_{2} \gamma_{3}+\alpha_{3} \gamma_{2}\right) .
\end{aligned}
$$

The conventional approach for evaluating the far field is to employ a steepest descent technique. For that purpose, it is convenient to introduce both the transformation

$$
h=k_{2} \sin w,
$$

which maps the $h$ plane onto a new complex plane $w=\sigma+i \eta$ and the elliptic cylindrical system of coordinates $r, \theta$ :

$$
\begin{aligned}
& z=\sqrt{\alpha_{2}} r \sin \theta, \\
& (l-x)={ }^{2} \varepsilon_{11} r \cos \theta .
\end{aligned}
$$

Then expression (6) is assumed in the form

$$
\begin{aligned}
& { }^{2} H_{y}(r, \theta)=\frac{\mathrm{i}}{4 \pi \sqrt{\alpha}} \int_{-\pi / 2+\mathrm{i} \infty}^{\pi / 2+\mathrm{i} \infty} q(c+d)^{-1} \exp \left\{\mathrm { i } k _ { 2 } \left[\alpha_{2} \cos (w+\theta)\right.\right. \\
& \left.\left.-{ }^{2} \varepsilon_{13} \cos \theta \sin w\right]\right\} \mathrm{d} w-\frac{1}{4 \pi \sqrt{\alpha_{2}}} \int_{-\pi / 2+\mathrm{i} \infty}^{\pi / 2+\mathrm{i} \infty} f(c+d)^{-1} \\
& \times \exp \left\{\mathrm{i} k_{2} r\left[\alpha_{2} \cos (w+\theta)-{ }^{2} \varepsilon_{13} \cos \theta \sin w\right]\right\} \mathrm{d} w .
\end{aligned}
$$

In the analysis of Eq. (16) one must now take into account the fact that the functions $q(c+d)^{-1}$ and $f(c+d)^{-1}$ are not unique as well as the fact that the $\theta$ angle is greater than the critical angle on the upper and lower walls of the waveguide

$$
\begin{aligned}
& \theta>\arcsin k_{1} / k_{2}, \\
& \theta>\arcsin k_{3} / k_{2} .
\end{aligned}
$$

To transform the double-valued functions $q(c+d)^{-1}$ and $f(c+d)^{-1}$ into the unique ones, it is neccessary to make the branch cuts $\Gamma_{1}$ and $\Gamma_{3}$ in the $w$-plane (Fig. 2). Each branch cut is drawn along contours on which

$$
\begin{aligned}
& \operatorname{Im}\left[\alpha_{1}\left(k_{1}^{2}-k_{2}^{2} \sin ^{2} w\right)\right]^{1 / 2}=0, \\
& \operatorname{Im}\left[\alpha_{3}\left(k_{3}^{2}-k_{2}^{2} \sin ^{2} w\right)\right]^{1 / 2}=0,
\end{aligned}
$$

respectively.

Deforming the integration path in Eq. (16) to the path of the steepest descent we meet both branch cuts $\Gamma_{1}$ and $\Gamma_{3}$ (Fig. 2.).

The use of the steepest descent technique leads to a branch cut contribution, if a branch point is crossed by the steepest descent path. The total integration path is shown in Fig. 2.

The steepest descent integration then yields

$$
{ }^{2} H_{y}={ }^{2} H_{\mathrm{ys}}+{ }^{2} H_{y \mathrm{~b}} \text {, }
$$




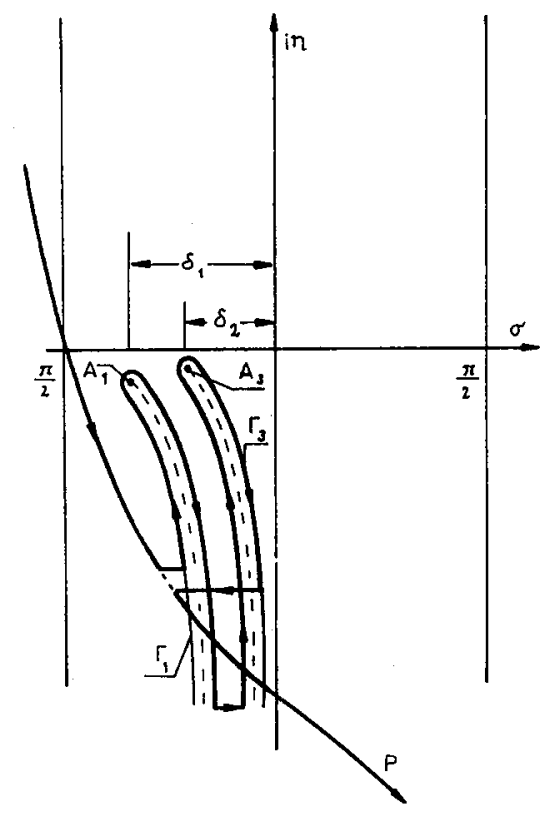

Fig. 2. Branch cuts and contour of integration in the $w$-plane.

where ${ }^{2} H_{y \mathrm{~s}}$ and ${ }^{2} H_{y \mathrm{~b}}$ refer, to the saddle point and the branch point contribution, respectively.

The lateral waves are given by integrals around branch cuts $\Gamma_{1}$ and $\Gamma_{3}$. For the $\Gamma_{1}$ contour the ${ }^{2} H_{y}$ component has the form

$$
\begin{aligned}
& { }^{2} H_{y}\left(\Gamma_{1}\right)=\frac{\mathrm{i}}{4 \pi \sqrt{\alpha_{2}}} \int_{-\mathrm{i} \infty}^{-A_{1}}+\Phi_{1}(w) \exp \left\{\mathrm{i} k_{2} \sqrt{\alpha_{2}} r\left[\cos (\theta+w)-{ }^{2} \varepsilon_{13} \cos \theta \sin w\right]\right\} \mathrm{d} w \\
& +\frac{\mathrm{i}}{4 \pi \sqrt{\alpha_{2}}} \int_{-A_{1}}^{-\mathrm{i} \infty}-\Phi_{1}(w) \exp \left\{\mathrm{i} k_{2} \sqrt{\alpha_{2}} r\left[\cos (\theta+w)-{ }^{2} \varepsilon_{13} \cos \theta \sin w\right]\right\} \mathrm{d} w \\
& -\frac{\mathrm{i}}{4 \pi \sqrt{\alpha_{2}}} \int_{-\mathrm{i} \infty}^{-A_{1}}+\Phi_{2}(w) \exp \left\{\mathrm{i} k_{2} \sqrt{\alpha_{2}} r\left[\cos (\theta+w)-{ }^{2} \varepsilon_{13} \cos \theta \sin w\right]\right\} \mathrm{d} w \\
& -\frac{\mathrm{i}}{4 \pi \sqrt{\alpha_{2}}} \int_{-A_{1}}^{-\mathrm{i} \infty}-\Phi_{2}(w) \exp \left\{\mathrm{i} k_{2} \sqrt{\alpha_{2}} r\left[\cos (\theta+w)-{ }^{2} \varepsilon_{13} \cos \theta \sin w\right]\right\} \mathrm{d} w, \quad(22)
\end{aligned}
$$

where $+\Phi_{1}(w)$ and $-\Phi_{1}(w)$ are the values of the reflection coefficients $q(c+d)^{-1}$ on the left and right hand side of the $\Gamma_{1}$ branch cut. ${ }^{+} \Phi_{2}(w)$ and $-\Phi_{2}(w)$ are the values of the reflection coefficients $f(c+d)^{-1}$ on the left and right hand side of the $\Gamma_{1}$ branch cut, $A_{1}$ is the branch point corresponding to the critical angle $\delta_{1}=\arcsin k_{1} / k_{2}$. 
In turn, for the $\Gamma_{3}$ contour we have

$$
\begin{aligned}
& { }^{2} H_{y}\left(\Gamma_{3}\right)=\frac{\mathrm{i}}{4 \pi \sqrt{\alpha_{2}}} \int_{-\mathrm{i} \infty}^{-A_{3}}+\Phi_{3}(w) \exp \left\{\mathrm{i} k_{2} \sqrt{\alpha_{2}} r\left[\cos (\theta+w)-{ }^{2} \varepsilon_{13} \cos \theta \sin w\right]\right\} \mathrm{d} w \\
& +\frac{\mathrm{i}}{4 \pi \sqrt{\alpha_{2}}} \int_{-A_{3}}^{-\mathrm{i} \infty}-\Phi_{3}(w) \exp \left\{\mathrm{i} k_{2} \sqrt{\alpha_{2}} r\left[\cos (\theta+w)-{ }^{2} \varepsilon_{13} \cos \theta \sin w\right]\right\} \mathrm{d} w \\
& -\frac{\mathrm{i}}{4 \pi \sqrt{\alpha_{2}}} \int_{-\mathrm{i} \infty}^{-A_{3}}+\Phi_{4}(w) \exp \left\{\mathrm{i} k_{2} \sqrt{\alpha_{2}} r\left[\cos (\theta+w)-{ }^{2} \varepsilon_{13} \cos \theta \sin w\right]\right\} \mathrm{d} w \\
& -\frac{\mathrm{i}}{4 \pi \sqrt{\alpha_{2}}} \int_{-A_{3}}^{-\mathrm{i} \infty}-\Phi_{4}(w) \exp \left\{\mathrm{i} k_{2} \sqrt{\alpha_{2}} r\left[\cos (\theta+w)-{ }^{2} \varepsilon_{13} \cos \theta \sin w\right]\right\} \mathrm{d} w, \quad(23)
\end{aligned}
$$

where ${ }^{+} \Phi_{3}(w)$ and $-\Phi_{3}(w)$ are the values of the reflection coefficient $q(c+d)^{-1}$ on the left and right hand side of the $\Gamma_{3}$ branch cut respectively, $+\Phi_{4}(w)$ and $-\Phi_{4}(w)$ are the values of the reflection coefficient $f(c+d)^{-1}$ on the left and right hand side of the $\Gamma_{3}$ branch cut and $A_{3}$ is the branch point corresponding to the critical angle $\delta_{2}=\arcsin k_{3} / k_{2}$.

We shall perform all our further calculations using the steepest descent method (see Appendix).

Thus we have asymptotic expression of (22):

$$
\begin{aligned}
& { }^{2} H_{y}\left(\Gamma_{1}\right) \sim \frac{\alpha_{2} \mathrm{e}^{-\mathrm{i} \pi / 4}}{\alpha_{1}^{2} k_{2}^{2}}\left(\frac{\alpha_{1} k_{1}}{2 \pi \cos \delta_{1}}\right)^{1 / 2} \frac{\alpha_{2} \gamma_{3}\left(\alpha_{2} \gamma_{3} \sin \Omega_{1}+\alpha_{3} \gamma_{2}{ }^{\prime} \cos \Omega_{1}\right)}{\left(\alpha_{3} \gamma_{2} \sin \Omega_{1}-\alpha_{2} \gamma_{3} \cos \Omega_{1}\right)^{2}} \\
& \times \frac{\exp \left[\mathrm{i} k_{2} r\left(\sqrt{\alpha_{2}} \cos \theta \cos \delta_{1}+\sqrt{\alpha_{2}} \sin \theta \sin \delta_{1}+{ }^{2} \varepsilon_{13} \cos \theta \sin \delta_{1}\right)\right]}{\left[r\left(\sqrt{\alpha_{2}} \sin \theta \cos \delta_{1}-\sqrt{\alpha_{2}} \cos \theta \sin \delta_{1}-{ }^{2} \varepsilon_{13} \cos \theta \cos \delta_{1}\right)\right]^{3 / 2}} \\
& \left.\times \exp \left(\mathrm{i} k_{2}{ }^{1} \varepsilon_{13} r_{0} \cos \theta_{0} \sin \delta_{1}\right)\right),
\end{aligned}
$$

where

$$
\begin{aligned}
& \Omega_{1}=2 l{ }^{2} \varepsilon_{11}^{-1}\left[\alpha_{2}\left(k_{2}^{2}-k_{1}^{2}\right)\right]^{1 / 2}, \\
& \dot{\gamma_{3}}=\left[\alpha_{3}\left(k_{1}^{2}-k_{3}^{2}\right)\right]^{1 / 2}, \\
& \gamma_{2}^{\prime}=\left[\alpha_{2}\left(k_{2}^{2}-k_{3}^{2}\right)\right]^{1 / 2}, \\
& r_{0} \cos \theta_{0}={ }^{2} \varepsilon_{11}^{-1}\left(l-x_{0}\right) .
\end{aligned}
$$

The asymptotic expression of (23):

$$
\begin{aligned}
& { }^{2} H_{y}\left(\Gamma_{3}\right) \sim \frac{\mathrm{i} \alpha_{2} \mathrm{e}^{-\mathrm{i} \pi / 4}}{\alpha_{3} k_{2}}\left(\frac{\alpha_{3} k_{3} \cos \delta_{2}}{2 \pi \alpha_{2}}\right)^{1 / 2} \frac{\left.\alpha_{1} \gamma_{2}{ }^{\prime} \cos \Omega_{2}+\alpha_{2} \gamma_{1} \sin \Omega_{2}\right)}{\left(\alpha_{1} \gamma_{2}{ }^{\prime} \sin \Omega_{2}-\alpha_{2} \gamma_{1} \cos \Omega_{1}\right)^{2}} \\
& \times \frac{\exp \left[\mathrm{i} k_{2} r\left(\sqrt{\alpha_{2}} \cos \theta \cos \delta_{2}+\sqrt{\alpha_{2}} \sin \theta \sin \delta_{2}+{ }^{2} \varepsilon_{13} \cos \theta \sin \delta_{2}\right)\right]}{\left[r\left(\sqrt{\alpha_{2}} \sin \theta \cos \delta_{2}-\sqrt{\alpha_{2}} \cos \theta \sin \delta_{2}-{ }^{2} \varepsilon_{13} \cos \theta \cos \delta_{2}\right)\right]^{3 / 2}} \\
& \times \exp \left[r_{0} \cos \theta_{0}\left(\gamma_{1}+\mathrm{i} k_{3}{ }^{1} \varepsilon_{13}\right)\right],
\end{aligned}
$$


where

$$
\begin{aligned}
& \Omega_{2}=2 l^{2} \varepsilon_{11}^{-1}\left[\alpha_{2}\left(k_{2}^{2}-k_{3}^{2}\right)\right]^{1 / 2} \\
& \gamma_{1}=\left[\alpha_{1}\left(k_{3}^{2}-k_{1}^{2}\right)\right]^{1 / 2}
\end{aligned}
$$

Relations (24) and (25) constitute the final forms for the lateral waves in the uniaxial optical waveguide.

\section{Interpretation of the obtained results}

The lateral waves (24) and (25) can be presented in a form which is convenient for interpretation. To do this, we shall introduce the following designations (see Fig. 3 and 4):
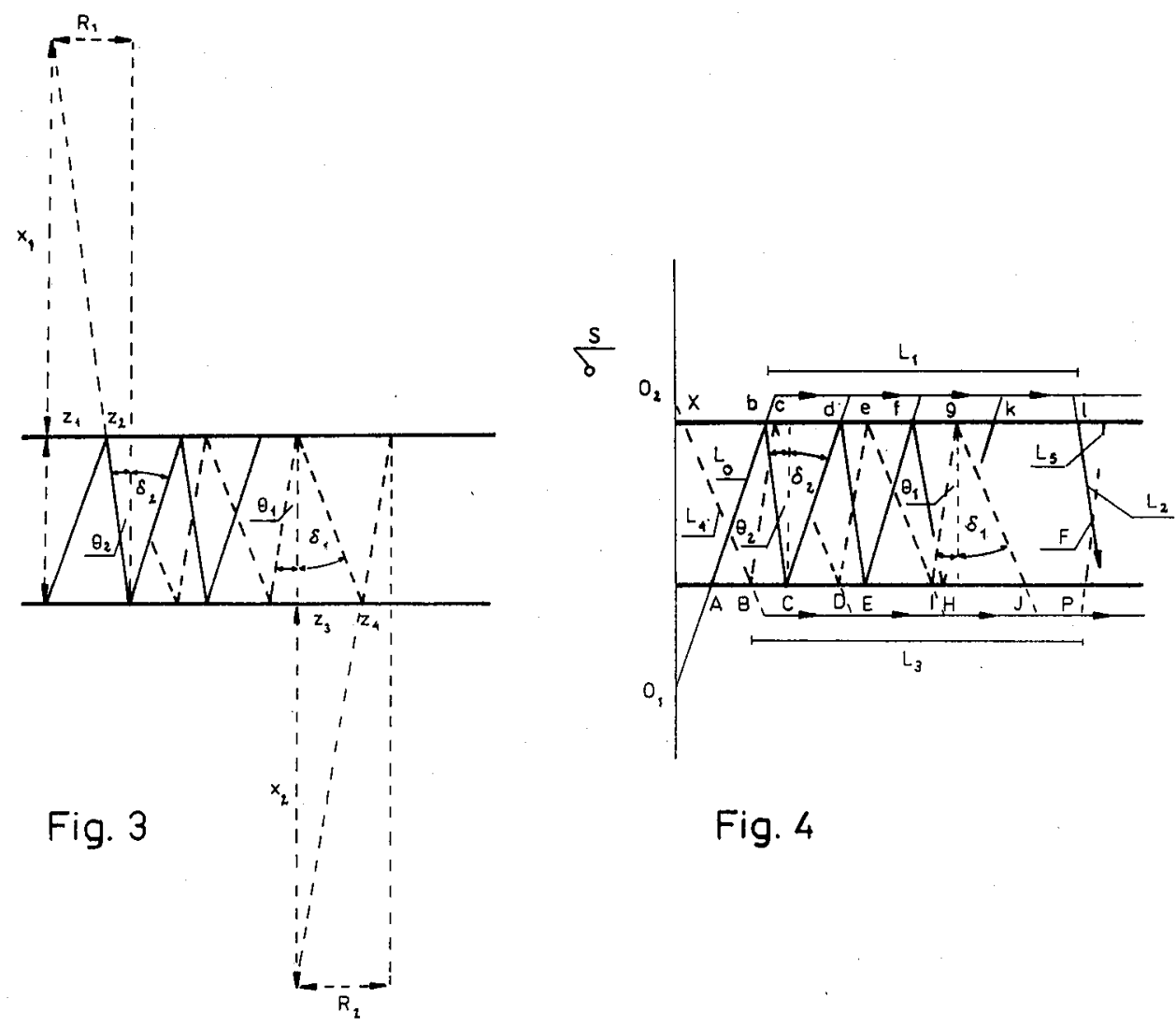

Fig. 4

Fig. 3. The denotations and symbols useful in the interpretation of the lateral waves.

Fig. 4. Lateral waves for uniaxial asymmetric waveguide. 


$$
\begin{aligned}
& r \sin \theta_{1}=R_{1}, \\
& x_{1}+2 l=r \cos \theta_{1}, \\
& x_{1}=\tan \delta_{1} / \tan \theta_{1},
\end{aligned}
$$

and

$$
\begin{aligned}
& r \sin \theta_{2}=R_{2}, \\
& x_{2}+2 l=r \cos \theta_{2}, \\
& x_{2}=2 l \frac{\tan \delta_{2}}{\tan \theta_{2}}, \\
& R_{1}=z_{1}+L_{1}+z_{2}, \\
& R_{2}=z_{3}+L_{3}+z_{4} .
\end{aligned}
$$

Making use of Eqs. (29)-(33), after some manipulations we obtain from (24) and (25) the following expressions for the lateral waves:

$$
\begin{aligned}
& H_{y}\left(\Gamma_{1}\right)=-\alpha_{2}^{2} \gamma_{3} k_{2}^{-1} \mathrm{e}^{-\mathrm{i} \pi / 4}\left(\frac{k_{1}}{2 \pi \alpha_{1}^{3} \cos \delta_{1}}\right)^{1 / 2} \frac{\left(\alpha_{2} \gamma_{3} \sin \Omega_{1}+\alpha_{3} \gamma_{2}{ }^{\prime} \cos \Omega_{1}\right)}{\left(\alpha_{3} \gamma_{2} \sin \Omega_{1}-\alpha_{2} \gamma_{3} \cos \Omega_{1}\right)^{2}} \\
& \times \frac{\exp \left[\mathrm{i}\left(\sqrt{\alpha_{2}} k_{1} L_{1}+\sqrt{\alpha_{2}} k_{2}^{-1} A_{1} B_{1} \sin ^{-1} \theta_{1}+{ }^{2} \varepsilon_{13} A_{1} k_{1} k_{2}^{-1} \cot \theta_{1}\right)\right]}{\left[\sqrt{\alpha_{2}} L_{1}\left(k_{2}^{2}-k_{1}^{2}\right)^{1 / 2}+\sqrt{\alpha_{2}} A_{1} C_{1} k_{2}^{-1} \sin ^{-1} \theta_{1}+{ }^{2} \varepsilon_{13} A_{1} k_{2}^{-1}\left(k_{2}^{2}-k_{1}^{2}\right)^{1 / 2} \cot \theta_{1}\right]^{3 / 2}} \\
& \times \exp \left(\mathrm{i} k_{2}{ }^{1} \varepsilon_{13} r_{0} \cos \theta_{0} \sin \delta_{1}\right),
\end{aligned}
$$

where

$$
\begin{aligned}
& A_{1}=k_{1} L_{0}+k_{2} L_{2} \sin \theta_{1}, \\
& B_{1}=k_{1} \sin \theta_{1}+\left(k_{2}^{2}-k_{1}^{2}\right)^{1 / 2} \cos \theta_{1}, \\
& C_{1}=\left(k_{2}^{2}-k_{1}^{2}\right)^{1 / 2} \sin \theta_{1}-k_{1} \cos \theta_{1},
\end{aligned}
$$

and

$$
\begin{aligned}
& H_{y}\left(\Gamma_{3}\right)=\mathrm{i} \alpha_{3}^{-1} \mathrm{e}^{-\mathrm{i} \pi / 4}\left(\frac{\alpha_{3} k_{3} \cos \delta_{1}}{2 \pi \alpha_{2}}\right)^{1 / 2} \frac{\left(\alpha_{1} \gamma_{1} \sin \Omega_{2}+\alpha_{1} \gamma_{2}{ }^{\prime} \cos \Omega_{2}\right)}{\left(\alpha_{1} \gamma_{2} \sin \Omega_{2}-\alpha_{2} \gamma_{1} \cos \Omega_{2}\right)^{2}} \\
& \times \frac{\exp \left[\mathrm{i}\left(\sqrt{\alpha_{2}} k_{3} L_{3}+\sqrt{\alpha_{2}} A_{2} B_{2} k_{2}^{-1} \sin ^{-1} \theta_{2}+{ }^{2} \varepsilon_{13} A_{2} k_{2}^{-1} \cot \theta_{2}\right)\right]}{\left[\sqrt{\alpha_{2}} L_{3}\left(k_{2}^{2}-k_{3}^{2}\right)^{1 / 2}+\sqrt{\alpha_{2}} A_{2} C_{2} k_{2}^{-1} \sin ^{-1} \theta_{2}+{ }^{2} \varepsilon_{13} A_{2} k_{2}^{-1}\left(k_{2}^{2}-k_{3}^{2}\right)^{1 / 2} \cot \theta_{2}\right]^{3 / 2}} \\
& \times \exp \left[r_{0} \cos \theta_{0}\left(\gamma_{1}+\mathrm{i} k_{3}{ }^{1} \varepsilon_{13}\right)\right],
\end{aligned}
$$

with

$$
\begin{aligned}
& A_{2}=k_{3} L_{4}+k_{2} L_{5} \sin \theta_{2}, \\
& B_{2}=k_{3} \sin \theta_{2}+\left(k_{2}^{2}-k_{3}^{2}\right)^{1 / 2} \cos \theta_{2}, \\
& C_{2}=\left(k_{2}^{2}-k_{3}^{2}\right)^{1 / 2} \sin \theta_{2}-k_{3} \cos \theta_{2} .
\end{aligned}
$$

The meaning of the designations $L_{0}, L_{1}, \ldots, L_{5}$ is illustrated in Fig. 4. 
The lateral wave is a field variety that is intimately associated with the wave undergoing total reflection when incidence occurs from the denser medium onto the interface to the less dense medium. This field is most strongly excited, if the incoming inhomogeneous field is incident at or very close to the critical angle of total reflection, i.e. $\theta_{1}=\delta_{1}$ or $\theta_{2}=\delta_{2}$. The lateral wave appears also in the case of the incidence angle greater than critical one. For $\theta_{1}>\delta_{1}$ or $\theta_{2}>\delta_{2}$ the incident wave penetrates into the less dense medium and travels some distance inside it, after which it reemerges in the denser medium. Consequently, the actual reflected energy flux is laterally displaced with respect to the reflected wave (Goos-Hanchen shift [2]). To examine some of the properties of the lateral wave, reference is made to Fig. 3 and Fig. 4.

We observe that two lateral waves may propagate independently along two interfaces of the waveguide. The wave (31) emerges at one of the image points $O_{1}$ at the critical angle $\delta_{1}$ and then travels to the field point $F$ along the path $O_{1} A b l F$ as shown in Fig. 4.

The wave (35) emerges at one of the image points $\mathrm{O}_{2}$ at the critical angle $\delta_{2}$ and travels to the field point $F$ along the path $O X B P F$. It is also possible to interpret the lateral waves as being produced directly by the actual source rather than the image source. This is shown in Fig. 4 by means of the path $S X A b C d E f H k l F$ or $S X B c D e I g J P F$ wherein three reflections at the slab interfaces are accounted.

The total internal reflection on the interface between uniaxial media is not a mirror reflection $[11,12]$. Generally, the reflection angle differs from the angle of incidence, i.e. $\theta_{1} \neq \delta_{1}$ and $\theta_{2} \neq \delta_{2}$ (Fig. 4).

This creates a situation in which the guidance of lateral waves is possible through both interfaces in the waveguide. Different waves which leave a real source can impinge on the upper interface at different angles. As a result of the refraction one of these waves can impinge further at the critical angle on the lower interface creating a lateral wave which is guided by the lower plane $x=-l$. Another wave due to the refraction can impinge on the lower interface at an angle greater than the critical one. These waves undergo the total reflection and can impinge on the upper interface at the critical angle. As a result, a lateral wave guided by the plane $x=l$ is created.

In an asymmetric structure of the waveguide the appropriate boundary conditions enable the lateral waves to propagate along each of the interfaces in such a way that the waves in the cover and the substrate vanish exponentially. The energy of the lateral waves does not leave the layer, but is confined within and guided by the film.

Because the wave number for the wave propagated in the cover is $k_{1}$ and in the substrate is $k_{3}$, the waves in the film can adjust themself to these conditions only when the propagation direction of the wave is at angle $\delta_{1}$ formed with the normal to the upper interface angle, and at angle $\delta_{2}$ with the normal to the lower interface angle such that

$$
\begin{aligned}
& k_{2} \sin \delta_{1}=k_{1}, \\
& k_{2} \sin \delta_{2}=k_{3},
\end{aligned}
$$

where $k_{2}$ is the wave number of the wave in the film. 
Conditions (39) and (40) are fulfilled by the lateral waves in the waveguide because these are at the same time conditions defining the critical angles (17) and (18). The above two conditions are satisfied in a waveguide simultaneously, because the continuity conditions on interfaces are fulfilled simultaneously.

All above-stated properties of the two lateral waves guided by the waveguide are characteristic of the surface wave guided by the waveguide. Thus the guidance of lateral waves by both interfaces in the waveguide is equivalent to the guidance of the surface wave.

The phenomenon of transformation of lateral waves into surface waves looks even more interesting if we realise that the lateral wave guided by the single interface is given by a suitable branch cut and belongs to a set of the radiation field modes (the continuous spectrum), while the surface wave is given by the residue (the pole) and belongs to a set of the discrete modes.

The boundary conditions in the waveguide can be chosen in such a way that the wave incident upon the first interface at the critical angle can impinge on the second one at an angle smaller than the critical one. Then each of the interfaces guides a lateral wave, but, at the same time, these two interfaces irradiate energy outside the layer. In this case the waveguide does not support a surface wave and the energy of the lateral waves is irradiated.

We can now notice that the amplitude of $H_{y}\left(\Gamma_{1}\right)$ is:

$$
\begin{aligned}
& H_{y}\left(\Gamma_{1}\right) \propto\left[\sqrt{\alpha_{2}} L_{1}\left(k_{2}^{2}-k_{1}^{2}\right)^{1 / 2}+\sqrt{\alpha_{2}} A_{1} C_{1} k_{2}^{-1} \sin ^{-1} \theta_{1}\right. \\
& \left.+{ }^{2} \varepsilon_{13} A_{1} k_{2}^{-1}\left(k_{2}^{2}-k_{1}^{2}\right)^{1 / 2} \cot \theta_{1}\right]^{-3 / 2} .
\end{aligned}
$$

While the geometric-optical field contributions decrease as $(k r)^{-1 / 2}$, the amplitude of lateral waves (34) and (38) decreases as $(k r)^{-3 / 2}$.

The presence of small losses in the waveguide causes the exponential decay of both the lateral waves and the geometric-optical field contributions. In this case the lateral waves decay much less rapidly than the geometric-optical field and at large distances from the source they are the dominant field in the waveguide.

We can notice that the lateral wave discussed above differs also from the lateral wave supported by the simplest possible boundary configuration in which the amplitude decreases as $\left(k_{1} L_{1}\right)^{-3 / 2}$ or $\left(k_{3} L_{3}\right)^{-3 / 2}$ only.

\section{The zigzag propagation model for an uniaxial optical waveguide}

The boundary conditions determine many unusual properties of the wave motion when one is considering the problem of the electromagnetic field propagation. The conditions which have to be fulfilled by the propagating field in the waveguide lead first of all to the spacial quantization of this field. Thus, by analogy with a resonant cavity the waveguide leads to the propagation of the field expressed by a set of discrete modes. Each mode is eigenfunction of the wave equation and the eigenvalues prove to be discrete and are the solutions of the corresponding equation for eigenvalues. Due to the two-dimensional geometry of planar waveguides the field distribution in the film can be represented by a superposition of two plane waves incident at an angle which is equal to or greater 
than the critical one. These waves propagate along a broken line and therefore are called the "zigzag" ones $[13,14]$.

Representing each of these plane waves by the corresponding ray or wave vector, the electromagnetic problem of propagation of the field in the waveguide can be easily transformed to a geometrical model which is popular in the literature $[8,9,13,14]$.

The spectral theory of the electromagnetic field and the implying "zigzag" propagation model presented here eliminate all those disadvantages. This theory finds out that the magnetic field in the film leading the field (region 2) is a sum of two components (21):

$$
{ }^{2} H_{y}={ }^{2} H_{y \mathrm{~s}}+{ }^{2} H_{y \mathrm{~b}} .
$$

The first component ${ }^{2} H_{y}$ represents the field to which the contribution are saddle points met by the path of steep descent during the integration of the expressions for the electromagnetic field (16). There are two such saddle points - one for each integral in (16).

Because the field yielded by the saddle points is the field of the geometrical optics, therefore the component ${ }^{2} H_{y}$ represents two geometric-optical waves falling on the upper and lower plane of the interface at the critical angles $\delta_{1}$ and $\delta_{2}$, respectively. At the interface each of these waves undergoes the reflection according to the principles of the geometrical optics and then superposes with the field represented by the second component of (42), i.e. with ${ }^{2} H_{y \mathrm{~b}}$.

As it is seen from Eq. (42) the contributions of ${ }^{2} H_{y \mathrm{~b}}$ are two branch cuts $\Gamma_{1}$ and $\Gamma_{2}$ (Fig. 2) met by the integration path in (16) in the representation of the "saddle point". The ${ }^{2} H_{y b}$ component represents thus two waves which become to be called as lateral waves.

Each of lateral waves propagates in the medium with the smaller refraction coefficient (the cover and the substrate) along the interface; from the point at which the wave vector of the reflected wave cuts the interface to the region of the geometrical shadow of the reflected wave. The analytical form for the lateral waves are determined by expressions (24) and (25).

The above-mentioned coupling between the geometric-optical wave and the lateral one is a very crucial point in the "zigzag" propagation model based on the spectral representation of the electromagnetic field in the optical waveguide.

Thus a geometric-optical wave incident on the interface at the angle which is critical or greater than the critical one gives rise to a combination of waves - the reflected and the lateral ones. The same combination of waves appears on the other interface in the waveguide when the geometric-optical wave falls at the critical angle or greater one.

The geometric-optical wave may be represented here by the wave reflected from the former interface and now incident at an angle greater from the critical one or any other wave from the source incident at the critical angle. The "zigzag" model in the approach of the spectral field theory is thus formed essentially by two waves: the geometric-optical and the lateral ones whereas the "driving mechanism" of the model is the phenomenon of the total internal reflection. In more strict terms the "zigzag" model in the presented theory includes one more spectral component 
of the field - the leaky waves. Their contributions are complex poles within the region comprised by the integration path in the "saddle point" representation.

Inhomogeneous waves, which are the leaky ones, fulfill the boundary conditions in the optical waveguide and constitute an independent component of the electromagnetic field. Undergoing only a partial reflection on the interface these waves propagate also in a zigzag manner. However, the energy of these waves is dissipated into the cover and the substrate of the waveguide. Therefore the leaky waves "escape" rapidly from the waveguide and do not constitute the constant component of the field propagation model. All we spoke above about the "zigzag" propagation model is valid both for isotropic as well as for anisotropic wa veguides. However, there exist also essential differences between the zigzag waves in both types of waveguides.

In contrast to the case of the isotropic medium, each component of the zigzag wave in the uniaxial planar waveguide has different wave number in the direction perpendicular to the waveguide axis ( $z$-axis). Because in the $z$-direction the wave numbers of modes are the same, we obtain, as a result, different wave numbers and different directions of wave vectors for both components. In consequence, the zigzag wave is not mirror symmetrical one with respect to the reflection occurring on the interfaces in the waveguide (the reflection angle is different from the incidence angle $[11,12])$. The geometrical illustration of the "zigzag" propagation model for the uniaxial planar waveguide is shown in Fig. 4.

The "zigzag" propagation model based on the spectral representation of the field stresses the important role of the lateral wave. This important role is particularly visible when the waveguide is multimodal and when there can appear modes connected with impinged waves on the interfaces at the critical angles or at angles close to critical ones. Another important case can be found for a lossy waveguide. In these type of waveguides both the space waves and the lateral waves are exponentially damped with the distance from the source. Let us note, therefore, that the main contribution to the field arises from the lateral waves which decay much less rapidly than the space waves. At far distances the lateral wave is the dominant one in the lossy waveguides. If the waveguide is composed of unlossy cover and substrate media and of a lossy medium of the film, then the lateral wave also forms the principal picture of the field in the waveguide. The field in the film is then quickly weakened, whereas the lateral wave passing most of its way in the substrate or cover does not undergo weakening, thus it is not the only component of the field.

\section{Conclusions}

The lateral waves in the uniaxial optical waveguide are closely associated with the phenomenon of the total reflection and arise due to the presence of branch point singularities in the reflection coefficients. In the structure of uniaxial asymmetric waveguides two sets of the lateral waves may propagate along two interfaces of the waveguide.

Two different situations connected with the guidance of lateral waves are realized. In the first situation two lateral waves are guided so that the field in the 
cover and the substrate vanish exponentially. The energy of the lateral waves is totally comprised in the film and the waveguide supports surface waves. In the second situation each of the interfaces guides the lateral wave, but at the same time the energy is radiated. In this case the waveguide does not support the surface wave.

The diffractional effects in the waveguide are related to the Goose-Hanchen shift which is caused by lateral waves.

The lateral wave concept, therefore, has to be taken into consideration in the process of electromagnetic field propagation in the waveguide, i.e. in the "zigzag" propagation model.

The above presented model of the field propagation in the uniaxial optical waveguide contains the zigzag waves formed from the superposition of the geometric-optical waves and two lateral waves of the (34) and (38) types. In contrast to the isotropic medium, both components of the zigzag wave are described by different wave numbers in the direction perpendicular to the waveguide $z$-axis. Because in the $z$-direction the wave numbers of modes are the same, we obtain, as a result, different wave numbers and different directions of wave vectors for both components. In consequence, the zigzag wave is not mirror symmetrical one with respect to the reflection on the waveguide boundaries.

\section{Appendix}

In this appendix we give some details on the theoretical treatment leading to the asymptotic expressions (24) and (25).

We deform the integration path in such a way that it passes from the branch point $A_{n}(n=1,3)$ over the steepest descent line

$$
\operatorname{Re}\left[\sqrt{\alpha_{2}} \cos (w+\theta)-{ }^{2} \varepsilon_{13} \cos \theta \sin w\right]=\text { const. }
$$

Substituting $w=\delta_{1}$ in (A1) and taking into account that $w=-\sigma-\mathrm{i} \eta$ (Fig. 2), from the last equation we obtain the relation for the integration path corresponding to the $\Gamma_{1}$ branch cut

$$
\begin{aligned}
& \sqrt{\alpha_{2}} \cos \theta \cos \sigma \operatorname{ch} \eta+\sqrt{\alpha_{2}} \sin \theta \sin \sigma \operatorname{ch} \eta+{ }^{2} \varepsilon_{13} \cos \theta \sin \sigma \operatorname{ch} \eta \\
& =\sqrt{\alpha_{2}} \cos \theta \cos \sigma_{1}-\sqrt{\alpha_{2}} \sin \theta \sin \delta_{1}-{ }^{2} \varepsilon_{13} \cos \theta \sin \delta_{1} .
\end{aligned}
$$

In the steepest descent method small values of $\eta$ play an essential role. For this reason we can put $\sigma=\delta_{1}, \operatorname{sh} \eta=\eta, \operatorname{ch} \eta=1, \mathrm{~d} w=\mathrm{i} \mathrm{d} \eta$. Taking this into account and performing the integration of (22) along the path of the steepest descent, after some single transformations we obtain the asymptotic expression given by (24).

The steepest descent path for integral (23) is given by the equation

$\sqrt{\alpha_{2}} \cos \theta \cos \sigma \operatorname{ch} \eta+\sqrt{\alpha_{2}} \sin \theta \sin \sigma \operatorname{ch} \eta+{ }^{2} \varepsilon_{13} \cos \theta \operatorname{ch} \eta$

$=\sqrt{\alpha_{2}} \cos \theta \cos \delta_{2}-\sqrt{\alpha_{2}} \sin \theta \sin \delta_{2}-{ }^{2} \varepsilon_{13} \cos \theta \sin \delta_{2}$.

Analogously to the latter case, performing the integration of (23) over the path given in (A3) we obtain asymptotic expression (25). 


\section{Acknowledgments}

The author expresses his gratitude to Prof. J. Petykiewicz and to Dr. K. Gniadek for many helpful discussions connected with this research.

\section{References}

[1] T. Tamir, Optik 36, 209 (1972).

[2] T. Tamir, Optik 37, 204 (1973).

[3] T. Tamir, Optik 38, 297 (1973).

[4] T. Tamir, A.A. Oliner, Proc. IEE 110, 310 (1963).

[5] T. Tamir, L.B. Felsen, IEEE Trans. Antennas Propag. 13, 410 (1965).

[6] R.E. Collin, Field Theory of Guided Waves, Mc Graw-Hill, New York 1960.

[7] L.B. Felsen, N. Marcuvitz, Radiation and Scattering of Waves, Prentice-Hall, New York 1973.

[8] I.M. Brekhovskich, Waves in Layered Media, Academic Press, New York 1960.

[9] D.P. Gia Russo, J.H. Harris, J. Opt. Soc. Am. 63, 138 (1973).

[10] M. Wabia, K. Gniadek, Acta Phys. Pol. A54, 493 (1978).

[11] M. Wabia, Acta Phys. Pol. A78, 861 (1990).

[12] M. Wabia, Acta Phys. Pol. A78, 873 (1990).

[13] P.K. Tien, Rev. Mod. Phys. 49, 361 (1977).

[14] H. Kogelnik, in: Integrated Optics, Springer-Verlag, Berlin 1975, p. 19. 